\title{
Maternal and paternal depression and child mental health trajectories: evidence from the Avon Longitudinal Study of Parents and Children
}

\author{
Priya Rajyaguru, Alex S. F. Kwong, Elizabeth Braithwaite and Rebecca M. Pearson
}

\section{Background}

The relationships between offspring depression profiles across adolescence and different timings of parental depression during the perinatal period remain unknown.

\section{Aims}

To explore different timings of maternal and paternal perinatal depression in relation to patterns of change in offspring depressive mood over a 14 year period.

\section{Method}

Data were obtained from the Avon Longitudinal Study of Parents and Children (ALSPAC). Parental antenatal depression (ANTD) was assessed at 18 weeks gestation, and postnatal depression (PNTD) at 8 weeks postpartum. Population-averaged trajectories of offspring depressive symptoms were estimated using the Short Mood and Feelings Questionnaire (SMFQ) on nine occasions between 10 and 24 years of age.

\section{Results}

Full data were available for 5029 individuals. Offspring exposed to both timings of maternal depression had higher depressive symptoms across adolescence compared with offspring not exposed to ANTD or PNTD, characterised by higher depressive symptoms at age 16 (7.07 SMFQ points $(95 \% \mathrm{Cl}=6.19,7.95$; $P<0.001))$ and a greater rate of linear change $(0.698$ SMFQ points
$(95 \% \mathrm{Cl}=0.47,0.93 ; P=0.002))$. Isolated maternal ANTD and to a lesser extent PNTD were also both associated with higher depressive symptoms at age 16, yet isolated maternal PNTD showed greater evidence for an increased rate of linear change across adolescence. A similar pattern was observed for paternal ANTD and PNTD, although effect sizes were attenuated.

\section{Conclusions}

This study adds to the literature demonstrating that exposure to two timings of maternal depression (ANTD and PNTD) is strongly associated with greater offspring trajectories of depressive symptoms.

\section{Keywords}

ALSPAC; child depression; adolescent depression; maternal depression; paternal depression; trajectories.

\section{Copyright and usage}

(c) The Author(s), 2021. Published by Cambridge University Press on behalf of the Royal College of Psychiatrists. This is an Open Access article, distributed under the terms of the Creative Commons Attribution licence (http://creativecommons.org/ licenses/by/4.0/), which permits unrestricted re-use, distribution, and reproduction in any medium, provided the original work is properly cited.
There is evidence linking maternal antenatal depression (ANTD), maternal postnatal depression (PNTD) and, more recently, paternal PNTD to a range of child outcomes including emotional, behavioural, cognitive and physical health consequences. ${ }^{1}$ However, the underlying mechanisms remain largely unknown. One perspective is that different timings of parental mental illness (i.e. offspring exposure before and/or after birth) may be linked to some pathways more than others in relation to child outcomes (see Stein et $\mathrm{al}^{1}$ for a comprehensive overview). We hypothesise that a child exposed to more timings of parental depression is at greater, cumulative risk of depression. One may further hypothesise that such children will also be more vulnerable to genetic risk load when considering the intergenerational transmission of depression. However, those exposed to maternal ANTD only may experience a specific 'preprogrammed' effect owing to the in utero environment uniquely provided by mothers. There is growing evidence that exposure to prenatal maternal stress increases risk of offspring affective problems and emotional instability. ${ }^{2,3}$ By contrast, exposure to PNTD in either parent may reflect risk transmitted via altered parenting behaviour, and the overall effect may be similar with exposure to each parent owing to a shared parenting pathway. ${ }^{4}$ In addition, given existing epidemiological evidence of gender differences ${ }^{5}$ and animal evidence for a female fetal vulnerability to stress hormones in relation to maternal ANTD exposure (for an overview of the animal literature, see Kapoor et $\mathrm{al}^{6}$ ), it is possible that female offspring may be more vulnerable to the effects of maternal ANTD. The unique research design presented here allows us to explore patterns of data that could indicate different mechanisms by which the intergenerational transmission of depression may occur. The key hypothesis is that in addition to high genetic risk load (presence of ANTD and PNTD in either parent or both parents) and risk transmitted via environmental means, including altered parenting behaviour (PNTD in either parent or both parents), there will be additional biological risk from exposure to maternal ANTD via prenatal programming mechanisms. In addition, female offspring exposed to maternal ANTD may be at greater risk of depression than males exposed to maternal ANTD.

Thus far the literature has focused on specific time points for child outcomes, ranging from birth up to age $18,{ }^{7}$ yet depression is known to change across adolescence and adulthood, with differential risk factors associated with different patterns of depression. ${ }^{8}$ Recent studies using advanced methods have attempted to explore trajectories of offspring risk in relation to parental perinatal mental health (e.g. O'Donnell et $\mathrm{al}^{9}$ ). This provides an opportunity to explore the nature of offspring mental health outcomes and how this may change over different periods of development. However, such research has been limited by the number of exposure timings available (in relation to parental ANTD and PNTD), the use of parent-reported outcome measures only and the total number of outcome assessments available (e.g. O'Donnell et $\mathrm{al},{ }^{9}$ where available outcome data were limited up to age 13). Thus, the potential effects of parental perinatal depression on changes in offspring depression across childhood, adolescence and into adulthood remain unclear, as do the associations with overall levels of depression across this period. This study aimed to directly address this problem by exploring different timings of maternal and 
paternal depression (ANTD and PNTD, in isolation and combination) in relation to patterns of change in child, adolescent and early adult depressive mood. Using a large longitudinal cohort, we aimed to address the overall level of depression and rate of change in offspring depressive symptoms specifically.

\section{Method}

\section{Study sample}

This retrospective cohort study used data from the Avon Longitudinal Study of Parents and Children (ALSPAC), a birth cohort that recruited pregnant women residing in Avon, UK, with expected dates of delivery 1 April 1991 to 31 December $1992 .{ }^{10-12}$ The initial cohort consisted of 14062 live births; when the oldest child participants were approximately 7 years of age, the initial sample was bolstered with participants who had failed to join the study originally. As a result, for all analyses involving data from the age of 7 onwards, there was a total of 14901 children alive at 1 year of age. Ethical approval for the study was obtained from the ALSPAC Ethics and Law Committee and the Local Research Ethics Committees. Informed consent for the use of data collected via questionnaires and clinics was obtained from participants following the recommendations of the ALSPAC Ethics and Law Committee at the time.

The study website contains details of all the data that are available through a fully searchable data dictionary and variable search tool: http://www.bristol.ac.uk/alspac/researchers/our-data. ${ }^{13}$ In addition, some of the data were collected using REDCap (https:// projectredcap.org/resources/citations/). ${ }^{14,15}$

\section{Measures}

\section{Parental depression}

Symptoms of parental depression were measured using the wellestablished Edinburgh Postnatal Depression Scale (EPDS). ${ }^{16}$ The EPDS is a ten-item self-report depression questionnaire validated for use in the perinatal period because it avoids physical symptoms. ${ }^{16}$ It is also validated for use outside the perinatal period and in men. ${ }^{17-19}$ Scores of $>12$ have a high sensitivity and specificity (estimates vary between $0.60-0.96$ for sensitivity and $0.45-0.97$ for specificity ${ }^{19}$ in predicting clinically diagnosed major depressive disorder ${ }^{18-20}$ ). In this work, scores of $>12$ were used as a binary cut-off with regard to the presence of parental perinatal depressive disorder. ANTD in mothers and fathers was assessed at 18 weeks gestation, and PNTD in mothers and fathers was assessed at 8 weeks postpartum.

\section{Offspring depression}

Self-reported depressive symptoms were measured on nine occasions between ages 10 and 24 using the Short Mood and Feelings Questionnaire (SMFQ). ${ }^{21}$ The SMFQ is a 13 item questionnaire widely used in adolescents that measures the presence of depressive symptoms over the past 2 weeks. In this study, it was administered via postal questionnaire or in clinics. Each item is scored between 0 and 2, with a summed total score ranging between 0 and 26. In this work, the total summed score was used in the analyses. The SMFQ correlates strongly with clinical depression ${ }^{22,23}$ and has been used to explore trajectories of depressive symptoms in other studies. ${ }^{8,24,25}$ Descriptive information on the SMFQ can be found in Supplementary Table 1 available at https://doi.org/10.1192/bjo. 2021.959 .

\section{Confounding variables}

The following confounders were included based upon previous literature examining early social risk factors and trajectories of depressive symptoms: ${ }^{8,24-26}$ sex (coded as a dummy variable for being female $($ male $=0$; female $=1)$ ), maternal/paternal educational attainment at birth (A-level or higher versus $\mathrm{O}$-level versus less than O-level), parity (first-born versus second-born versus third-born or more) and maternal/paternal smoking in pregnancy (no versus yes). Maternal analyses were adjusted for current paternal depression, and paternal analyses were adjusted for current maternal depression.

\section{Analysis}

Trajectories of depressive symptoms were estimated using multilevel growth-curve modeling. ${ }^{27}$ Briefly, multilevel growth-curve models create population-averaged trajectories, with individual level trajectories varying around this population average (i.e. each person may have their own trajectory that deviates from the population average). Descriptive statistics and previous analysis of this data have shown that the change in depressive symptoms is nonlinear and fluctuates over adolescence and young adulthood. ${ }^{8}$ To model this non-linearity, a multilevel quartic growth-curve polynomial model was chosen, in line with previous research using higher-order multilevel growth-curve polynomials for examining trajectories of depressive symptoms. ${ }^{8}$ Age was grand-mean centred to 16.53 years (the mean age of all assessments) in order to improve interpretation, ${ }^{28}$ as the model intercept and intercept variance then corresponded to the middle of adolescence. Such models provide an estimate of depressive symptom scores at a given intercept age but also quantify how depressive symptoms change over time through linear, quadratic, cubic and quartic polynomial effects (slope or rate-of-change terms). In all analyses, the polynomial age terms were allowed to vary randomly across individuals to capture each individual's unique trajectory (i.e. a random intercept and slope model). Further information regarding model selection and model equations can be found in the Supplementary material.

We ran separate models to examine the association between maternal and paternal depression and offspring depressive symptom trajectories. The first model examined population-averaged trajectories for maternal depression, which was classified into four groups: no ANTD/no PNTD (baseline group), yes ANTD/no PNTD, no ANTD/yes PNTD and yes ANTD/yes PNTD. These groups were then interacted with the intercept and four polynomial terms to create four distinct trajectories of offspring depressive symptoms corresponding to the four groups. The same method was used for the paternal analysis. We also included interaction terms for gender and stratified analyses to run exploratory analyses on whether these effects differed by offspring sex.

All analyses were conducted using Stata $15^{29}$ with the userwritten runmlwin command, ${ }^{30}$ which calls the standalone multilevel modelling package MLwiN v3.01 (www.cmm.bristol.ac.uk/ MLwiN/index.shtml). The Stata code used in this analysis can be found at https://github.com/kwongsiufung.

\section{Missing data}

Missing data in the trajectories analysis were handled using full information maximum likelihood (FIML), which assumes that the data are missing at random. ${ }^{31}$ The FIML approach assumes that the probability of an individual missing a depressive symptom measure does not depend on their underlying depressive symptoms on that occasion, given their observed trajectory on other occasions. Previous analysis has used FIML to examine trajectories of depressive symptoms in ALSPAC and found that this method handles missing data well in trajectories analysis. ${ }^{8}$ To maximise power, we included individuals in this analysis if they had at least one measurement of depressive symptoms. 
Table 1 Participant demographics for maternal depression exposure

\begin{tabular}{|c|c|c|c|}
\hline & $\begin{array}{l}\text { Sample with complete exposure data } \\
\text { (maternal depression) }{ }^{\mathrm{a}}, N(\%)\end{array}$ & $\begin{array}{l}\text { Sample with complete outcome data (at least } \\
\text { one offspring depression) }{ }^{\mathrm{b}}, N(\%)\end{array}$ & $\begin{array}{l}\text { Sample with complete exposure, } \\
\text { outcome and confounding datac }, N(\%)\end{array}$ \\
\hline \multicolumn{4}{|l|}{ Sex } \\
\hline Male & $5369(51.41)$ & $4495(47.85)$ & $2447(48.66)$ \\
\hline Female & $5075(48.59)$ & 4899 (52.15) & $2582(51.34)$ \\
\hline \multicolumn{4}{|l|}{ Maternal education } \\
\hline A Level or higher & 3739 (36.94) & $3453(40.87)$ & $2814(44.04)$ \\
\hline GCSE/O Level & $6382(63.06)$ & $4996(59.13)$ & $2215(55.04)$ \\
\hline \multicolumn{4}{|l|}{ Parity } \\
\hline First-born & $4573(44.51)$ & $3918(45.94)$ & 2465 (49.02) \\
\hline Second-born & 3695 (35.96) & $3041(35.66)$ & 1745 (34.70) \\
\hline Third-born + & 2006 (19.53) & 1569 (18.40) & 819 (16.29) \\
\hline \multicolumn{4}{|l|}{ Smoking } \\
\hline No prenatal smoking & 8087 (77.43) & $6942(80.23)$ & $4202(83.56)$ \\
\hline Yes postnatal smoking & $2357(22.57)$ & $1711(18.77)$ & 827 (16.44) \\
\hline \multicolumn{4}{|l|}{ Paternal depression } \\
\hline No ANTD/no PNTD & $6413(94.48)$ & $5284(94.63)$ & 4770 (94.85) \\
\hline Yes ANTD/no PNTD & $156(2.30)$ & $121(2.17)$ & $103(2.05)$ \\
\hline No ANTD/yes PNTD & $141(2.08)$ & $115(2.06)$ & 99 (1.97) \\
\hline Yes ANTD/yes PNTD & 78 (1.15) & $64(1.15)$ & $57(1.13)$ \\
\hline \multicolumn{4}{|c|}{$\begin{array}{l}\text { ANTD, antenatal depression; PNTD, postnatal depression; SMFQ, Short Mood and Feelings Questionnaire; N, number of offspring. } \\
\text { a. Number of offspring for whom data on maternal perinatal depression were available. Reading down the exposure sample column, the figures also indicate the number of offspring who } \\
\text { had data available on each individual variable in addition to maternal depression information. } \\
\text { b. Number of offspring for whom outcome data of at least one SMFQ result were available. Reading down the outcome sample column, the figures also indicate the number of offspring who } \\
\text { had data available on each individual variable in addition to having at least one SMFQ result. } \\
\text { c. The number of offspring for whom data were available on maternal depression, confounding variables and at least one SMFQ result. }\end{array}$} \\
\hline
\end{tabular}

\section{Sensitivity analyses}

Sensitivity analyses using a more stringent threshold (only including individuals with at least four SMFQ time point measures rather than one) yielded similar results (Supplementary Fig. 1 and Table 2). As a further check to examine the effects of parental depression on offspring trajectories, we also included a later time point of parental depression as a covariate in our models when the offspring were roughly 22 years old, yielding similar findings (Supplementary Table 3).

\section{Results}

\section{Descriptive results}

Table 1 provides an overview of offspring demographics as per the exposure, outcome and final sample, and those excluded for the maternal analyses. Supplementary Table 4 outlines the offspring demographics for the paternal analyses. With respect to the primary outcome measure, the SMFQ reliability coefficient was stable $(\alpha=0.8-0.92)$ across the nine time points of offspring outcome assessment. For further descriptive information with regards to the SMFQ used in this study, please see Supplementary Table 1. The final analyses were composed of complete, adjusted data for 5029 offspring in the maternal analyses and 4534 offspring in the paternal analyses (Tables 2 and 4). For the unadjusted analyses, please see Supplementary Tables 5 and 6.

\section{Main results}

\section{Exposure to maternal ANTD and PNTD}

Using the no ANTD and no PNTD group as a reference (Fig. 1 and Table 2), exposure to both maternal ANTD and PNTD placed offspring in the highest risk group overall. The intercept score for the baseline group was 5.35 (95\% CI 5.14-5.62, $P<0.01$ ), and the linear rate of change was 0.33 ( $95 \% \mathrm{CI} 0.29-0.37, P<0.01)$. Thus, from here on, the $\beta^{\text {diff }}$ value refers to the difference in intercept and slope terms compared with this baseline group, and a higher $\beta^{\text {int }}$ value indicates the score of the comparator group (i.e. the higher risk groups). Having a mother with both ANTD and PNTD resulted in offspring SMFQ scores that were 1.72 points higher at age 16 $\left(\beta^{\text {diff }}=1.72,95 \% \mathrm{CI}=0.84-2.59, P<0.001\right)$, producing an intercept value of $7.07\left(\beta^{\text {int }}=7.07,95 \% \mathrm{CI}=6.19-7.95, P=<0.001\right)$, and the highest linear rate of change in depressive symptom scores per year ( $\left.\beta^{\text {int }}=0.698,95 \% \mathrm{CI}=0.47-0.93, P=0.002\right)$. In other words, in terms of predicted differences, the results shown in Table 3 indicate that by age 24 the maximum score difference between the two extreme groups was 2.89 points $(95 \% \mathrm{CI}=1.44-4.35, P<0.001)$. Additional rate of change parameters showed varying effects and are displayed in Table 2, with accompanying predicted differences in Table 3. Exposure to maternal ANTD but not PNTD also resulted in SMFQ scores that were 1.43 points higher at age $16\left(\beta^{\text {diff }}=1.43\right.$, 95\% $\mathrm{CI}=0.87-1.99, P<0.001)$, producing an intercept value of $6.78\left(\beta^{\text {int }}=6.78,95 \% \mathrm{CI}=6.22-7.35, P \leq 0.001\right)$; however, there was weak evidence for substantive change over time. In this group, compared with no exposure to either ANTD or PNTD, the difference in scores by age 24 was 1.90 points $(1.02-2.78, P<0.001)$. Finally, exposure to maternal PNTD alone (in the absence of ANTD) resulted in the smallest increase in SMFQ scores, with scores approximately 0.78 points higher at age $16\left(\beta^{\text {diff }}=0.78,95 \% \mathrm{CI}=0.11-1.44, P=0.02\right)$ and an intercept value of $6.13\left(\beta^{\text {int }}=6.13,95 \% \mathrm{CI}=5.47-6.79\right.$, $P=0.02)$. However, exposure to PNTD alone was also weakly associated with a higher linear rate of change in depressive symptom scores per year $\left(\beta^{\text {int }}=0.51,95 \% \mathrm{CI}=0.34-0.67, P=0.04\right)$.

\section{Exposure to paternal ANTD and PNTD}

Using the no ANTD and no PNTD group as a reference (see Fig. 1, where the intercept for this baseline group was 5.40 and the linear rate of change was 0.35 , and Table 4 ), it was clear that overall exposure to both paternal ANTD and PNTD placed offspring in the highest risk group, with SMFQ scores that were 1.37 points higher at age $16\left(\beta^{\text {diff }}=1.37,95 \% \mathrm{CI}=-0.15-2.89, P=0.08\right)$, producing an intercept of $6.77\left(\beta^{\text {int }}=6.77,95 \% \mathrm{CI}=5.25-8.296, P=0.08\right)$. In terms of predicted differences, the results shown in Table 5 demonstrate that by age 24 , the maximum score difference between the two groups was 2.63 (95\% CI $0.54-4.72, P=0.014$ ). However, exposure to either paternal ANTD alone or paternal PNTD alone showed little association with higher depressive symptoms. In terms of rate of change of depressive symptoms, there was little evidence of a difference across groups, although the trends were similar 

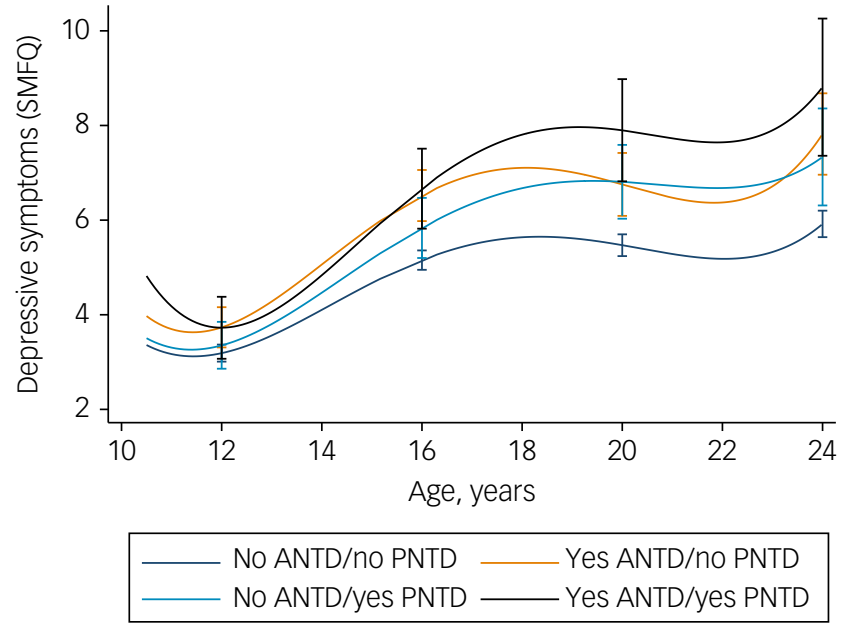

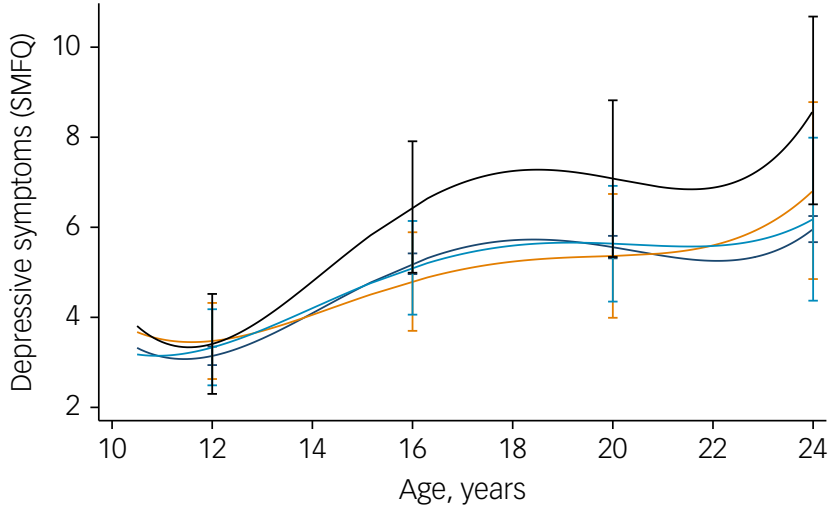

No ANTD/no PNTD No ANTD/yes PNTD
Yes ANTD/no PNTD Yes ANTD/yes PNTD

Fig. 1 Growth curves to demonstrate the different trajectories of offspring depressive symptom course, determined by the presence of maternal ANTD and/or PNTD exposure (left-hand side) and paternal ANTD and/or PNTD exposure (right-hand side). The $95 \%$ Cls are plotted at ages $12,16,20$ and 24 years.

\begin{tabular}{|c|c|c|c|c|c|c|c|c|}
\hline \multirow{2}{*}{$\begin{array}{l}\text { Parameter } \\
\text { No ANTD/no PNTD Intercept }\end{array}$} & \multirow{2}{*}{$\begin{array}{l}\text { Beta } \\
5.353\end{array}$} & \multicolumn{2}{|c|}{$95 \% \mathrm{Cls}$} & \multirow{2}{*}{$\begin{array}{l}\text { s.e. } \\
0.107\end{array}$} & \multirow{2}{*}{$\begin{array}{l}P \text {-value } \\
<0.001\end{array}$} & \multirow{2}{*}{$\begin{array}{c}\text { Baseline + risk group } \\
\text { score parameter } \\
-\end{array}$} & \multicolumn{2}{|c|}{$\begin{array}{l}95 \% \mathrm{Cl}+\text { risk group } \\
\text { score parameter }\end{array}$} \\
\hline & & 5.144 & 5.562 & & & & - & - \\
\hline No ANTD/no PNTD age ${ }^{a}$ & 0.328 & 0.290 & 0.366 & 0.020 & $<0.001$ & - & - & - \\
\hline No ANTD/no PNTD age^^2 $2^{b}$ & -0.088 & -0.099 & -0.076 & 0.006 & $<0.001$ & - & - & - \\
\hline No ANTD/no PNTD age $\wedge 3^{c}$ & -0.005 & -0.006 & -0.004 & 0.001 & $<0.001$ & - & - & - \\
\hline No ANTD/no PNTD age ${ }^{\wedge} 4^{d}$ & 0.002 & 0.001 & 0.002 & 0.0001 & $<0.001$ & - & - & - \\
\hline Yes ANTD/no PNTD intercept & 1.430 & 0.868 & 1.992 & 0.287 & $<0.001$ & 6.783 & 6.219 & 7.347 \\
\hline Yes ANTD/no PNTD age ${ }^{a}$ & 0.092 & -0.052 & 0.235 & 0.073 & 0.21 & 0.420 & 0.282 & 0.558 \\
\hline Yes ANTD/no PNTD age^2 $2^{b}$ & -0.046 & -0.091 & -0.001 & 0.023 & 0.046 & -0.133 & -0.177 & -0.090 \\
\hline Yes ANTTD/no PNTD age ${ }^{\wedge} 3^{c}$ & -0.001 & -0.005 & 0.003 & 0.002 & 0.593 & -0.006 & -0.009 & 0.002 \\
\hline Yes ANTD/no PNTD age^ $4^{d}$ & 0.001 & -0.00001 & 0.002 & 0.0005 & 0.054 & 0.002 & 0.00200 & 0.003 \\
\hline No ANTD/yes PNTD intercept & 0.776 & 0.114 & 1.439 & 0.338 & 0.022 & 6.130 & 5.466 & 6.793 \\
\hline No ANTD/yes PNTD age $e^{a}$ & 0.179 & 0.010 & 0.348 & 0.086 & 0.038 & 0.507 & 0.342 & 0.672 \\
\hline No ANTD/yes PNTD age^^2 $2^{b}$ & 0.002 & -0.051 & 0.054 & 0.027 & 0.953 & -0.086 & -0.137 & -0.035 \\
\hline No ANTD/yes PNTD age^^3 ${ }^{c}$ & -0.002 & -0.006 & 0.003 & 0.002 & 0.412 & -0.007 & -0.011 & -0.002 \\
\hline No ANTD/yes PNTD age ${ }^{\wedge} 4^{d}$ & 0.0000 & -0.001 & 0.001 & 0.001 & 0.989 & 0.002 & 0.001 & 0.003 \\
\hline Yes ANTD/yes PNTD intercept & 1.719 & 0.844 & 2.594 & 0.447 & $<0.001$ & 7.072 & 6.191 & 7.953 \\
\hline Yes ANTD/yes PNTD age ${ }^{a}$ & 0.370 & 0.140 & 0.601 & 0.117 & 0.002 & 0.698 & 0.471 & 0.925 \\
\hline Yes ANTD/yes PNTD age^^2 & -0.035 & -0.108 & 0.038 & 0.037 & 0.348 & -0.122 & -0.194 & -0.051 \\
\hline Yes ANTD/yes PNTD age^^3c & -0.008 & -0.014 & -0.002 & 0.003 & 0.01 & -0.013 & -0.018 & -0.007 \\
\hline Yes ANTD/yes PNTD age^ $4^{d}$ & 0.001 & -0.0003 & 0.003 & 0.001 & 0.124 & 0.003 & 0.0010 & 0.004 \\
\hline \multicolumn{9}{|c|}{$\begin{array}{l}\text { The No ANTD/no PNTD variable is the baseline (reference) group. There are four groups. Each group has an intercept and four terms resulting in four trajectories per group. The different } \\
\text { terms (a-d) for each group are further parameters to account for the non-linearity of the trajectories as seen by the fact that the slope is steeper at different ages. For further information on } \\
\text { the nature of change in rate of depressive symptoms as per the four terms at different ages, please see the Supplementary material. The intercept for each group was determined by } \\
\text { manually adding the baseline intercept for No ANTD/no PNTD to the intercept of the group being compared. Similarly, the rate of change for each subsequent group was determined by } \\
\text { adding the rate of change for the baseline group to the rate of change of the group being compared. This is highlighted in the column entitled 'Baseline + risk group score parameter'. For } \\
\text { ease of interpretation, we present the original regression coefficients and their } 95 \% \text { Cl, s.e. and } P \text {-values first. The s.e. and P-value represent the difference between the baseline trajectory } \\
\text { and the additional parameter. Then we present the scores for each group when the baseline group is added to the 'higher' risk group. This analysis was adjusted for sex, paternal depression, } \\
\text { maternal education at birth, parity and maternal smoking in pregnancy. Intercepts are centred to age 16, the mean age of all assessments. } \\
\text { ANTD, antenatal depression; PNTD, postnatal depression. } \\
\text { a. linear slope. } \\
\text { b. quadratic slope. } \\
\text { c. cubic change in speed of slope. } \\
\text { d. quartic slope. }\end{array}$} \\
\hline
\end{tabular}

to those observed in the maternal data (with rate of change following paternal PNTD alone being greater than that following paternal ANTD alone).

\section{Sex effects}

Overall, females had higher SMFQ scores at age 16 and faster rates of change in depressive symptom scores per year compared with male offspring (see Supplementary Figs 2 and 3 and Supplementary Tables 7 and 8 for further detail).
Exposure to maternal ANTD and PNTD by sex. Exposure to both maternal ANTD and PNTD placed female offspring in the highest risk group, resulting in SMFQ scores that were 3.997 points higher at age $16\left(\beta^{\text {int }}=8.48,95 \% \mathrm{CI}=7.35-9.62, P \leq 0.001\right)$ than those for male offspring not exposed at either time (Supplementary Table 7). Female offspring exposed to maternal ANTD only were also at increased risk $\left(\beta^{\text {int }}=7.92,95 \% \mathrm{CI}=7.22-8.63, P<0.001\right)$. Male offspring exposed at both times scored 1.73 points higher at age 16 $\left(\beta^{\text {int }}=6.21,95 \% \mathrm{CI}=4.90-7.52, P=0.01\right)$ than males with no exposure at either time. There was also some evidence suggesting that 
Table 3 Predicted differences in depressive symptoms scores at various ages between maternal antenatal depression (ANTD)/postnatal depression (PNTD) groups and offspring trajectories of depressive symptoms (DS)

\begin{tabular}{|c|c|c|c|c|c|}
\hline \multirow[t]{2}{*}{ Trajectories compared } & & \multicolumn{4}{|c|}{ Predicted mean difference $(95 \% \mathrm{Cl})$ in DS at various ages } \\
\hline & & 12 years & 16 years & 20 years & 24 years \\
\hline \multirow[t]{3}{*}{ No ANTD/no PNTD } & Yes ANTD/no PNTD & $\begin{array}{c}0.54(0.14,0.95) \\
P=0.009\end{array}$ & $\begin{array}{c}1.37(0.83,1.91) \\
P<0.001\end{array}$ & $\begin{array}{c}1.28(0.62,1.95) \\
P<0.001\end{array}$ & $\begin{array}{c}1.90(1.02,2.78) \\
P<0.001\end{array}$ \\
\hline & No ANTD/yes PNTD & $\begin{array}{c}0.16(-0.32,0.65) \\
P=0.509\end{array}$ & $\begin{array}{c}0.68(0.05,1.31) \\
P=0.035\end{array}$ & $\begin{array}{c}1.34(0.55,2.12) \\
P=0.001\end{array}$ & $\begin{array}{c}1.42(0.37,2.46) \\
P=0.008\end{array}$ \\
\hline & Yes ANTD/yes PNTD & $\begin{array}{c}0.54(-0.10,1.18) \\
P=0.101\end{array}$ & $\begin{array}{c}1.51(0.67,2.35) \\
P<0.001\end{array}$ & $\begin{array}{c}2.43(1.35,3.51) \\
P<0.001\end{array}$ & $\begin{array}{c}2.89(1.44,4.35) \\
P<0.001\end{array}$ \\
\hline \multirow[t]{2}{*}{ Yes ANTD/no PNTD } & No ANTD/yes PNTD & $\begin{array}{c}0.38(-0.23,0.99) \\
P=0.224\end{array}$ & $\begin{array}{c}0.69(-0.12,1.49) \\
P=0.094\end{array}$ & $\begin{array}{c}0.05(-0.94,1.05) \\
P=0.914\end{array}$ & $\begin{array}{c}0.49(-0.84,1.81) \\
P=0.472\end{array}$ \\
\hline & Yes ANTD/yes PNTD & $\begin{array}{c}0.01(-0.73,0.75) \\
P=0.987\end{array}$ & $\begin{array}{c}0.14(-0.83,1.12) \\
P=0.770\end{array}$ & $\begin{array}{c}1.14(-0.10,2.39) \\
P=0.072\end{array}$ & $\begin{array}{c}0.99(-0.67,2.66) \\
P=0.243\end{array}$ \\
\hline No ANTD/yes PNTD & Yes ANTD/yes PNTD & $\begin{array}{c}0.37(-0.41,1.16) \\
P=0.352\end{array}$ & $\begin{array}{c}0.83(-0.20,1.86) \\
P=0.113\end{array}$ & $\begin{array}{c}1.09(-0.22,2.40) \\
P=0.104\end{array}$ & $\begin{array}{c}1.48(-0.28,3.24) \\
P=0.100\end{array}$ \\
\hline
\end{tabular}

\section{Table 4 Paternal adjusted trajectories of offspring depressive symptoms $(N=4534)$}

\begin{tabular}{|c|c|c|c|c|c|c|c|c|}
\hline \multirow{2}{*}{$\begin{array}{l}\text { Parameter } \\
\text { No ANTD/no PNTD intercept }\end{array}$} & \multirow{2}{*}{$\begin{array}{l}\text { Beta } \\
5.402\end{array}$} & \multicolumn{2}{|c|}{$95 \% \mathrm{Cl}$} & \multirow{2}{*}{$\begin{array}{l}\text { s.e. } \\
0.118\end{array}$} & \multirow{2}{*}{$\begin{array}{l}P \text {-value } \\
<0.001\end{array}$} & \multirow{2}{*}{$\begin{array}{c}\text { Baseline + risk group score } \\
\text { parameter } \\
-\end{array}$} & \multicolumn{2}{|c|}{$\begin{array}{l}95 \% \mathrm{Cl}+\text { risk group } \\
\text { score parameter }\end{array}$} \\
\hline & & 5.171 & 5.632 & & & & - & - \\
\hline No ANTD/no PNTD age ${ }^{a}$ & 0.349 & 0.311 & 0.388 & 0.019 & $<0.001$ & - & - & - \\
\hline No ANTD/no PNTD age $\wedge 2^{b}$ & -0.090 & -0.101 & -0.078 & 0.006 & $<0.001$ & - & - & - \\
\hline No ANTD/no PNTD age $\wedge 3^{c}$ & -0.005 & -0.006 & -0.004 & 0.001 & $<0.001$ & - & - & - \\
\hline No ANTD/no PNTD age ${ }^{\wedge} 4^{d}$ & 0.002 & 0.001 & 0.002 & 0.0001 & $<0.001$ & - & - & - \\
\hline Yes ANTD/no PNTD intercept & -0.450 & -1.586 & 0.686 & 0.580 & 0.438 & 4.952 & 3.811 & 6.093 \\
\hline Yes ANTD/no PNTD age ${ }^{a}$ & -0.082 & -0.375 & 0.211 & 0.150 & 0.582 & 0.267 & -0.023 & 0.558 \\
\hline Yes ANTD/no PNTD age^^2 $2^{\mathrm{b}}$ & 0.042 & -0.053 & 0.137 & 0.048 & 0.391 & -0.048 & -0.142 & 0.046 \\
\hline Yes ANTD/no PNTD age^^3c & 0.003 & -0.005 & 0.010 & 0.004 & 0.498 & -0.003 & -0.010 & 0.005 \\
\hline Yes ANTD/no PNTD age^^ $4^{d}$ & -0.0005 & -0.002 & 0.001 & 0.001 & 0.625 & 0.001 & -0.001 & 0.003 \\
\hline No ANTD/yes PNTD intercept & -0.121 & -1.195 & 0.952 & 0.548 & 0.825 & 5.280 & 4.202 & 6.359 \\
\hline No ANTD/yes PNTD age ${ }^{a}$ & -0.044 & -0.328 & 0.239 & 0.145 & 0.759 & 0.305 & 0.024 & 0.586 \\
\hline No ANTD/yes PNTD age^^ $2^{b}$ & 0.029 & -0.059 & 0.117 & 0.045 & 0.524 & -0.061 & -0.148 & 0.026 \\
\hline 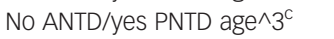 & 0.002 & -0.005 & 0.010 & 0.004 & 0.528 & -0.003 & -0.010 & 0.005 \\
\hline No ANTD/yes PNTD age $4^{d}$ & -0.001 & -0.002 & 0.001 & 0.001 & 0.505 & 0.001 & -0.001 & 0.003 \\
\hline Yes ANTD/yes PNTD intercept & 1.370 & -0.150 & 2.890 & 0.775 & 0.077 & 6.772 & 5.247 & 8.296 \\
\hline Yes ANTD/yes PNTD age ${ }^{a}$ & 0.180 & -0.198 & 0.558 & 0.193 & 0.351 & 0.529 & 0.153 & 0.906 \\
\hline Yes ANTD/yes PNTD age^^ $2^{b}$ & -0.045 & -0.167 & 0.078 & 0.063 & 0.476 & -0.134 & -0.256 & -0.012 \\
\hline Yes ANTD/yes PNTD age $3^{c}$ & -0.002 & -0.012 & 0.008 & 0.005 & 0.691 & -0.007 & -0.017 & 0.003 \\
\hline Yes ANTD/yes PNTD age^ $4^{d}$ & 0.001 & -0.001 & 0.003 & 0.001 & 0.397 & 0.003 & 0.0001 & 0.005 \\
\hline \multicolumn{9}{|c|}{$\begin{array}{l}\text { The No ANTD/no PNTD variable is the baseline (reference) group. There are four groups. Each group has an intercept and four terms resulting in four trajectories per group. The different } \\
\text { terms (a-d) for each group are further parameters to account for the non-linearity of the trajectories as seen by the fact that the slope is steeper at different ages. For further information on } \\
\text { the nature of change in rate of depressive symptoms as per the four terms at different ages, please see the Supplementary material. The intercept for each group was determined by } \\
\text { manually adding the baseline intercept for No ANTD/no PNTD to the intercept of the group being compared. Similarly, the rate of change for each subsequent group was determined by } \\
\text { adding the rate of change for the baseline group to the rate of change of the group being compared. This is highlighted in the column entitled 'baseline + risk group score parameter'. For } \\
\text { ease of interpretation, we present the original regression coefficients and their } 95 \% \text { Cl, s.e. and P-values first. The s.e. and P-value represent the difference between the baseline trajectory } \\
\text { and the additional parameter. Then we present the scores for each group when the baseline group is added to the 'higher' risk group. This analysis was adjusted for sex, maternal } \\
\text { depression, paternal education at birth, parity and paternal smoking in pregnancy. Intercepts are centred to age 16, the mean age of all assessments. } \\
\text { ANTD, antenatal depression; PNTD, postnatal depression. } \\
\text { a. linear slope. } \\
\text { b. quadratic slope. } \\
\text { C. cubic change in speed of slope. } \\
\text { d. quartic slope. }\end{array}$} \\
\hline
\end{tabular}

male offspring exposed to maternal ANTD only $\left(\beta^{\text {int }}=6.13,95 \% \mathrm{CI}\right.$ $=5.28-6.98, P<0.001)$ were also at risk. Linear rate of change in depressive symptoms scores was greater for females exposed to both maternal ANTD and PNTD $\left(\beta^{\text {int }}=0.61,95 \% \mathrm{CI}=0.30-0.91\right.$, $P=0.04)$ compared with males of non-depressed parents. Female offspring exposed to maternal PNTD only demonstrated an increase in rate of change of $0.58 \mathrm{SMFQ}$ points $\left(\beta^{\text {int }}=0.58,95 \% \mathrm{CI}=0.36-0.795\right.$, $P=0.008)$ at each time point. By contrast, a weaker association was seen for rate of change of depressive symptoms for female offspring exposed to maternal ANTD only. For males, a similar trend was observed, with those exposed to both maternal ANTD and PNTD demonstrating greater linear rate of change $\left(\beta^{\text {int }}=0.75,95 \%\right.$ $\mathrm{CI}=0.41-1.09, P=0.007)$ than any other male group.
Exposure to paternal ANTD and PNTD by sex. In relation to fathers (Supplementary Table 8), exposure to both paternal ANTD and PNTD placed female offspring in the highest risk group, with greater depressive symptoms at age $16\left(\beta^{\text {int }}=9.02,95 \% \mathrm{CI}=6.99\right.$ $11.05, P \leq 0.001)$. This was followed by exposure to paternal ANTD alone, which produced an increase in depressive symptom scores at age 16 among female offspring $\left(\beta^{\text {int }}=7.65,95 \% \mathrm{CI}=\right.$ 5.93-9.37, $P \leq 0.01$ ). Exposure to paternal PNTD alone was also associated with increased depressive symptom scores in female offspring but not to the same degree $\left(\beta^{\text {int }}=6.62,95 \% \mathrm{CI}=5.17-8.07\right.$, $P=0.005)$. Associations regarding the rate of change in depressive symptom scores in females were weak in comparison with the maternal estimates. With regards to male offspring, exposure to different 
Table 5 Predicted differences in depressive symptoms scores at various ages between paternal antenatal depression (ANTD)/postnatal depression (PNTD) groups and offspring trajectories of depressive symptoms (DS)

\begin{tabular}{|c|c|c|c|c|c|}
\hline \multirow[t]{2}{*}{ Trajectories compared } & & \multicolumn{4}{|c|}{ Predicted mean difference $(95 \% \mathrm{Cl}$ ) in DS at various ages } \\
\hline & & 12 & 16 & 20 & 24 \\
\hline \multirow[t]{3}{*}{ No ANTD/no PNTD } & Yes ANTD/no PNTD & $\begin{array}{c}0.33(-0.50,1.17) \\
P=0.437\end{array}$ & $\begin{array}{c}0.39(-0.69,1.48) \\
P=0.477\end{array}$ & $\begin{array}{c}0.20(-1.18,1.57) \\
P=0.781\end{array}$ & $\begin{array}{c}0.85(-1.11,2.82), \\
P=0.395\end{array}$ \\
\hline & No ANTD/yes PNTD & $\begin{array}{c}0.19(-0.65,1.02), \\
P=0.658\end{array}$ & $\begin{array}{c}0.09(-0.94,1.12) \\
P=0.864\end{array}$ & $\begin{array}{c}0.08(-1.20,1.36) \\
P=0.904\end{array}$ & $\begin{array}{c}0.22(-1.59,2.03) \\
P=0.811\end{array}$ \\
\hline & Yes ANTD/yes PNTD & $\begin{array}{c}0.27(-0.84,1.37) \\
P=0.636\end{array}$ & $\begin{array}{c}1.26(-0.19,2.72) \\
P=0.090\end{array}$ & $\begin{array}{c}1.52(-0.22,3.27) \\
P=0.086\end{array}$ & $\begin{array}{c}2.63(0.54,4.72) \\
P=0.014\end{array}$ \\
\hline \multirow[t]{2}{*}{ Yes ANTD/no PNTD } & No ANTD/yes PNTD & $\begin{array}{c}0.14(-1.03,1.31) \\
P=0.809\end{array}$ & $\begin{array}{c}0.30(-1.79,1.18) \\
P=0.687\end{array}$ & $\begin{array}{c}0.27(-2.14,1.59) \\
P=0.774\end{array}$ & $\begin{array}{c}0.63(-2.02,3.29), \\
P=0.640\end{array}$ \\
\hline & Yes ANTD/yes PNTD & $\begin{array}{c}0.07(-1.30,1.44) \\
P=0.987\end{array}$ & $\begin{array}{c}1.66(-0.15,3.46) \\
P=0.072\end{array}$ & $\begin{array}{c}1.72(-0.48,3.92) \\
P=0.126\end{array}$ & $\begin{array}{c}1.78(-1.07,4.63), \\
P=0.222\end{array}$ \\
\hline No ANTD/yes PNTD & Yes ANTD/yes PNTD & $\begin{array}{c}0.08(-1.29,1.45) \\
P=0.911\end{array}$ & $\begin{array}{c}1.35(-0.42,3.12) \\
P=0.135\end{array}$ & $\begin{array}{c}1.45(-0.70,3.59), \\
P=0.187\end{array}$ & $\begin{array}{c}2.41(-0.34,5.16), \\
P=0.085\end{array}$ \\
\hline
\end{tabular}

timings of paternal depression followed a similar trend as those observed for females, yet associations were much weaker in relation to depressive symptoms at age 16 or rate of change in symptoms.

\section{Discussion}

There is a growing body of evidence linking different time points of parental depression to different child mental health outcomes. ${ }^{7,9}$ However, these have typically been in relation to a specific offspring time point. To the best of our knowledge, this study is the first to explore the potential associations of different timings of maternal and paternal depression with offspring depressive symptoms over an extended period of childhood, adolescence and young adulthood using growth-curve analyses. We explored the overall level of offspring depression in terms of an intercept point and the rate of change in depressive symptoms (as demonstrated by the slope of the growth curve). We explored patterns of data in the context of several proposed (and not mutually exclusive) mechanisms; specifically, that ANTD in the mother may be linked genetically but also via possible fetal programming pathways facilitated by the placenta. By contrast, although ANTD in fathers may contribute equally to a genetic effect, it would not share mechanisms via the placenta. ${ }^{1,7,9}$ We also explored the nature of the associations with PNTD, which may relate more to ongoing rates of depression in offspring due to continued exposure of parental symptoms over the course of development.

\section{Strengths and limitations}

Strengths of this study include the comparison of two perinatal depression exposure time points (ANTD and PNTD) in mothers and fathers, thus building on previous research which has been limited by only single time points of parental perinatal depression being available. This allowed us to explore several potential pathways in the intergenerational transmission of depression. Further strengths include the longitudinal analysis over a 14 year period of childhood, adolescence and early adulthood, and the selfreported measure of offspring depressive symptoms. To the best of our knowledge, this is also the first study to explore offspring depression trajectories in relation to timings of parental perinatal depression in this way. However, limitations include the low numbers of fathers in the sample, which limited the interpretation of the associated paternal results. In addition, although we used FIML to adjust for missing data, this may represent a source of bias if the data were not missing at random. Moreover, given the observational nature of this study's design, it was not possible to infer causality or explicitly demonstrate the interpretations made.

\section{Scientific implications and mechanisms}

The literature exploring the potential mechanisms linking parental perinatal depressive symptoms to child outcomes considers several possibilities crossing both the antenatal and postnatal time periods. Genetics, prenatal in utero exposure, disruption to parent-child attachment, emotion regulation, modelling, family dysfunction and parenting ${ }^{32}$ have all been suggested. Of these possibilities, some are potentially specific to mothers and the antenatal period, including in utero or fetal programming effects often attributed to maternal biological changes affecting offspring brain development. ${ }^{33}$ However, definitive conclusions in relation to the fetal programming of offspring depression remains unknown, and further empirical evidence is required. ${ }^{34,35}$ Beyond the antenatal period, additional exposures may occur postnatally (i.e. from parenting, modelling, family dysfunction) and together with genetic effects may result from either parent.

In this study, we found that the accumulation of exposure to both timings of ANTD and PNTD, from mothers and fathers, carried the greatest risk for offspring. This finding is particularly relevant as it suggests that it is the accumulation of different exposure timings (as opposed to one exposure timing over another) that has the greatest influence on offspring depression course later in life. This increased risk, as seen for the maternal ANTD- and PNTD-exposed offspring group, may reflect the potential influence of combined antenatal mechanisms and subsequent environmental exposure with later maternal depression (i.e. transmission through multiple pathways). It is particularly noteworthy that following maternal exposure, we found the maximum difference in offspring depressive symptom scores between the two extreme groups to be 2.89 points at age 24 years. This corresponds to a 0.5 standard deviation and $11 \%$ increase in depressive symptoms, which is a larger difference than that which is found in recovery following depression treatment and thus could be considered clinically relevant. ${ }^{34,35}$ These findings add to the existing literature (including studies that have undertaken trajectory analyses) which has historically focused on only one specific time point. ${ }^{36-40}$

In relation to isolated maternal ANTD, there was some evidence for the specificity of effects, with exposed offspring having higher depressive symptom scores at age 16 compared with paternal ANTD or PNTD in either parent. This may be suggestive of further and specific maternal pathways heightening the risk following exposure to maternal ANTD. One such possibility is a fetal 
programming mechanism which may confer additional risk on top of genetic and environmental or social mechanisms. Some evidence for such a unique ANTD mechanism was also seen when exploring outcome by gender. It was clear that female offspring appeared to be at greatest risk overall, with higher depressive symptom scores than males across a variety of exposures, but especially so in relation to ANTD exposure. Although there were clear associations between maternal and paternal ANTD and female depression, it is possible that the underlying mechanisms involved are different. For example, there is accumulating evidence that female offspring are more vulnerable to prenatal maternal 'stress', and that this effect may be mediated by glucocorticoid mechanisms. ${ }^{41,42}$ However, given the lack of direct physiological connection to the fetus in utero, paternal ANTD associated risk is more likely to reflect a shared genetic liability. However, we also found some evidence that male offspring exposed to maternal ANTD (but not maternal PNTD) were at increased risk. These findings differ from previous research, which has found maternal ANTD to be associated with depression in females but not males. ${ }^{43-45}$ These findings, if replicated, may suggest an aspect of male offspring risk that has not been previously appreciated.

We also found evidence suggesting that exposure to maternal PNTD alone (as opposed to maternal ANTD alone) was associated with a greater rate of change in depressive symptom scores in offspring. This effect was directly observed when comparing the ANTD-only maternal analysis with the PNTD-only maternal analysis. In addition, we noted that although exposure to maternal ANTD alone was associated with greater depressive symptoms at age 16 (but not a heightened rate of change), the effect on the linear rate of change in depressive symptoms was not seen until inclusion of maternal PNTD (presence of both maternal ANTD and PNTD), which further suggests a role of maternal PNTD in relation to the linear rate of change in depressive symptoms. This effect was not seen in those offspring exposed to paternal depression, although as previously mentioned this may have been due to the low power of the analysis. From a pragmatic perspective, however, these findings could be explained by the potential effects of postnatal depression on parental emotions, cognitions and behaviour. These in turn have been linked to parenting, which is considered to be one of the most important mediators on the pathway leading to intergenerational transmission of mental health. ${ }^{1}$

\section{Clinical implications}

The results suggested in this paper highlight that young people exposed to both parental ANTD and PNTD may represent a vulnerable group. We also observed a dynamic effect of maternal PNTD on the rate of change of depressive symptoms in offspring, potentially highlighting a role for the development and delivery of interventions seeking to buffer the effects of parental mental illness during childhood and adolescence.

\section{Interpretation}

This study found that offspring exposed to both parental ANTD and PNTD were at greatest risk of depressive symptoms. We also found some evidence that could be consistent with the idea that although different timings of parental perinatal depression share some risk mechanisms, there may be additional and different ANTD pathways involved. Finally, we observed a specific effect of maternal PNTD exposure on the rate of change of offspring depressive symptoms over time. However, these interpretations need to be directly tested, and replication with larger numbers is required, in particular, for the paternal analyses.
Priya Rajyaguru (D. MBBCh. MRCPsych, Centre for Academic Mental Health, University of Bristol, Bristol, and Oxford Health NHS Foundation Trust, UK; Alex S. F. Kwong (D), of Bristol, Bristol, and Oxford Health NHS Foundation Trust, UK; Alex S. F. Kwong $\mathbb{~}$,
PhD, Population Health Sciences, Bristol Medical School, and Medical Research Council Integrative Epidemiology Unit, University of Bristol, Bristol, UK; Elizabeth Braithwaite, PhD, Department of Psychology, School of Health, Psychology and Social Care, Manchester Metropolitan University, Manchester, UK; Rebecca M. Pearson, PhD, Population Health Sciences, Bristol Medical School, and Medical Research Council Integrative Epidemiology Unit, University of Bristol, and National Institute for Health Research Bristol Biomedical Research Centre, Bristol, UK

Correspondence: Priya Rajyaguru. Email: priya.rajyaguru@bristol.ac.uk

First received 6 Feb 2020, final revision 21 May 2021, accepted 7 Jun 2021

\section{Supplementary material}

Supplementary material is available online at https://doi.org/10.1192/bjo.2021.959.

\section{Data availability}

P.R., A.S.F.K. and R.M.P. had access to the study data, and access is currently ongoing. The data were obtained from ALSPAC. Access to these data can be requested through application to the ALSPAC executive via the online proposal system (http://www.bristol.ac.uk/alspac/researchers/access/)

\section{Acknowledgements}

We thank all the families who took part in this study, the midwives for their help in recruiting them, and the whole ALSPAC team, which includes interviewers, computer and laboratory technicians, clerical workers, research scientists, volunteers, managers, receptionists and technicises.

\section{Author contributions}

P.R., A.S.F.K., E.B. and R.M.P. met the ICMJE criteria for authorship.

\section{Funding}

The UK Medical Research Council, the Wellcome Trust (grant 102215/2/13/2), and the University of Bristol provided core support for the ALSPAC. A comprehensive list of grants funding is availof Bristol provided core support for the ALSPAC. A comprehensive list of grants funding is avail-
able on the ALSPAC website (http://www.bristol.ac.uk/alspac/external/documents/grantacknowledgements.pdf). This study was also supported by the National Institute for Health Research Biomedical Research Centre at the University Hospitals Bristol National Health Service Foundation Trust and the University of Bristol, the National Institutes of Health (grant R01 DK10324) and the European Research Council under the European Union's Seventh Framework Programme (grant FP/2007-2013). This specific work was supported by a European Research Council Grant Agreement (grants 758813; Mental Health Intergenerational Transmission 'MHINT') held by R.M.P. and providing a salary for A.S.F.K. At the time of writing, P.R. was an ST6 Child and Adolescent Psychiatry Registrar employed by Oxford Health NHS Foundation Trust. A.S.F.K. is supported by an Economic Social Research Council Postdoctoral Fellowship (grant ES/V011650/1).

\section{Declaration of interest}

\section{References}

1 Stein A, Pearson RM, Goodman SH, Rapa E, Rahman A, McCallum M, et al. Effects of perinatal mental disorders on the fetus and child. Lancet 2014; 384 (9956): 1800-19.

2 Hill J, Pickles A, Wright N, Braithwaite E, Sharp H. Predictions of children's emotionality from evolutionary and epigenetic hypotheses. Sci Rep 2019; 9(1): 1-11.

3 Braithwaite E, Murphy S, Ramchandani P. Prenatal risk factors for depression: a critical review of the evidence and potential mechanisms. J Dev Origins Health Dis 2014; 5(5): 339-50.

4 Gutierrez-Galve L, Stein A, Hanington L, Heron J, Lewis G, O'Farrelly C, et al. Association of maternal and paternal depression in the postnatal period with offspring depression at age 18 years. JAMA Psychiatry 2019; 76(3): 290-6.

5 Quarini C, Pearson RM, Stein A, Ramchandani PG, Lewis G, Evans J. Are female children more vulnerable to the long-term effects of maternal depression during pregnancy? J Affect Disord 2016; 189: 329-35.

6 Kapoor A, Dunn E, Kostaki A, Andrews MH, Matthews SG. Fetal programming of hypothalamo-pituitary-adrenal function: prenatal stress and glucocorticoids. J Physiol 2006; 572: 31-44.

7 Pearson RM, Evans J, Kounali D, Lewis G, Heron J, Ramchandani PG, et al. Maternal depression during pregnancy and the postnatal period: risks and possible mechanisms for offspring depression at age 18 years. JAMA Psychiatry 2013; 70(12): 1312-9. 
8 Kwong ASF, Manley D, Timpson NJ, Pearson RM, Heron J, Sallis $\mathrm{H}$, et al. Identifying critical points of trajectories of depressive symptoms from child hood to young adulthood. J Youth Adolesc 2019; 48(4): 815-27.

9 O'Donnell KJ, Glover V, Barker ED, O'Connor TG. The persisting effect of maternal mood in pregnancy on childhood psychopathology. Dev Psychopathol 2014; 26(2): 393-403.

10 Boyd A, Golding J, Macleod J, Lawlor DA, Fraser A, Henderson J, et al. Cohort profile: the 'children of the $90 \mathrm{~s}^{\prime}$-the index offspring of the Avon longitudinal study of parents and children. Int J Epidemiol 2013; 42(1): 111-27.

11 Fraser A, Macdonald-Wallis C, Tilling K, Boyd A, Golding J, Davey Smith G, et al Cohort profile: the avon longitudinal study of parents and children: ALSPAC mothers cohort. Int J Epidemiol 2013; 42(1): 97-110.

12 Northstone K, Lewcock M, Groom A, Boyd A, Macleod J, Timpson NJ, et al. The Avon Longitudinal Study of Parents and Children (ALSPAC): an updated on the enrolled sample of index children in 2019. Wellcome Open Res 2019; 4: 51.

13 University of Bristol. ALSPAC Data Dictionary and Variable Search Tool. University of Bristol (http://www.bristol.ac.uk/alspac/researchers/our-data/ [cited 14 Oct 2020])

14 Harris PA, Taylor R, Thielke R, Payne J, Gonzalez N, Conde JG. Research electronic data capture (redcap)—a metadata-driven methodology and workflow process for providing translational research informatics support. J Biomed Inform 2009; 42(2): 377-81.

15 Harris PA, Taylor R, Minor BL, Elliott V, Fernandez M, O'Neal L, et al. The REDCap consortium: building an international community of software platform partners. J Biomed Inform 2019; 95: 103208

16 Cox JL, Holden JM, Sagovsky R. Detection of postnatal depression: development of the 10-item edinburgh postnatal depression scale. $\mathrm{Br} J$ Psychiatry 1987; 150(6): 782-6.

17 Edmondson OJH, Psychogiou L, Vlachos H, Netsi E, Ramchandani PG. Depression in fathers in the postnatal period: assessment of the Edinburgh postnata depression scale as a screening measure. J Affect Disord 2010; 125(1-3): 365-8.

18 Shakespeare J. Evaluation of Screening for Postnatal Depression against the NSC Handbook Criteria. National Screening Committee, 2001

19 Hewitt C, Gilbody S, Brealey S, Paulden M, Palmer S, Mann R, et al. Methods to identify postnatal depression in primary care: an integrated evidence synthesis and value of information analysis. Health Technol Assess 2009; 13(36): 1-145.

20 Gaynes BN, Gavin N, Meltzer-Brody S, Lohr KN, Swinson T, Gartlehner G, et al Perinatal depression: prevalence, screening accuracy, and screening outcomes. Evid Rep Technol Assess (Summ) 2005; 119: 1-8.

21 Angold A, Costello EJ, Messer SC, Pickles A. Development of a short questionnaire for use in epidemiological studies of depression in children and adolescents. Int J Methods Psychiatr Res 1995; 5(4): 237-49.

22 Thapar A, McGuffin P. Validity of the shortened Mood and Feelings Questionnaire in a community sample of children and adolescents: a preliminary research note. Psychiatry Res 1998; 81: 259-68.

23 Turner N, Joinson C, Peters TJ, Wiles N, Lewis G. Validity of the short mood and feelings questionnaire in late adolescence. Psychol Assess 2014. Available from: https://doi.org/10.1037/a0036572.supp.

24 Mahedy L, Hammerton G, Teyhan A, Edwards AC, Kendler KS, Moore SC, et al. Parental alcohol use and risk of behavioral and emotional problems in offspring. PLOS ONE 2017; 12(6): e0178862.

25 Rice F, Riglin L, Thapar AK, Heron J, Anney R, O'Donovan MC, et al Characterizing developmental trajectories and the role of neuropsychiatric genetic risk variants in early-onset depression. JAMA Psychiatry 2018. Available from: https://doi.org/10.1001/jamapsychiatry.2018.3338.

26 Kingsbury M, Weeks M, MacKinnon N, Evans J, Mahedy L, Dykxhoorn J, et al. Stressful life events during pregnancy and offspring depression: evidence from a prospective cohort study. J Am Acad Child Adolesc Psychiatry 2016; 55(8): 709-16e2.
27 Bryk AS, Raudenbush SW. Hierachical Linear Models for Social and Behavioral Research: Application and Data Analysis Methods. Sage, 1987.

28 Rawana JS, Morgan AS. Trajectories of depressive symptoms from adolescence to young adulthood: the role of self-esteem and body-related predictors. $J$ Youth Adolesc 2014; 43(4): 597-611.

29 StataCorp. Stata Statistical Software: Release 15. StataCorp LLC, 2017.

30 Leckie G, Charlton C. runmlwin: a program to run the MLwiN multilvel modeling software from within stata. J Stat Softw 2012; 52(11).

31 Little RJA, Rubin DB. Statistical Analysis with Missing Data (3rd edn). John Wiley \& Sons, 2020.

32 Goodman SH, Gotlib IH. Risk for psychopathology in the children of depressed mothers: a developmental model for understanding mechanisms of transmission. Psychol Rev 1999; 106(3): 458-90.

33 Glover V, O'Connor TG, O'Donnell K. Prenatal stress and the programming of the HPA axis. Neurosci Biobehav Rev 2010; 35(1): 17-22.

34 Lewis G, et al. The clinical effectiveness of sertraline in primary care and the role of depression severity and duration (PANDA): a pragmatic, double-blind, placebo-controlled randomised trial. Lancet Psychiatry 2019; 6(1): 903-14.

35 Carpenter JK, et al. Cognitive behavioral therapy for anxiety and related disorders: a meta-analysis of randomized placebo-controlled trials. Depress Anxiety 2018: 35(6): 502-14.

36 Fernandez Castelao C, Kroner-Herwig B. Different trajectories of depressive symptoms in children and adolescents: predictors and differences in girls and boys. J Youth Adolesc 2013; 42(8): 1169-82.

37 Yaroslavsky I, Pettit JW, Lewinsohn PM, Seeley JR, Roberts RE. Heterogeneous trajectories of depressive symptoms: adolescent predictors and adult outcomes. J Affect Disord 2013; 148(2-3): 391-9.

38 Mazza JJ, Fleming CB, Abbott RD, Haggerty KP, Catalano RF. Identifying trajectories of adolescents' depressive phenomena: an examination of early risk factors. J Youth Adolesc 2010; 39(6): 579-93.

39 Weeks M, Cairney J, Wild TC, Ploubidis GB, Naicker K, Colman I. Early-life predictors of internalizing symptom trajectories in Canadian children. Depress Anxiety 2014; 31(7): 608-16.

40 Whalen DJ, Luby JL, Tilman R, Mike A, Barch D, Belden AC. Latent class profiles of depressive symptoms from early to middle childhood: predictors, outcomes, and gender effects. J Child Psychol Psychiatry 2016; 57(7): 794-804.

41 Van den Bergh BR, Van Calster B, Smits T, Van Huffel S, Lagae L. Antenata maternal anxiety is related to HPA-axis dysregulation and self-reported depressive symptoms in adolescence: a prospective study on the fetal origins of depressed mood. Neuropsychopharmacology 2008; 33(3): 536-45.

42 de Bruijn AT, van Bakel HJ, Wijnen H, Pop VJ, van Baar AL. Prenatal maternal emotional complaints are associated with cortisol responses in toddler and preschool aged girls. Dev Psychobiol 2009; 51(7): 553-63.

43 Van den Bergh BR, Van Calster B, Smits T, Van Huffel S, Lagae L. Antenatal maternal anxiety is related to hpa-axis dysregulation and self-reported depressive symptoms in adolescence: a prospective study on the fetal origins of depressed mood. Neuropsychopharmacology 2008; 33(3): 536-45.

44 Costello EJ, Worthman C, Erkanli A, Angold A. Prediction from low birth weight to female adolescent depression: a test of competing hypotheses. Arch Gen Psychiatry 2007; 64(3): 338-44.

45 Van Lieshout RJ, Boylan K. Increased depressive symptoms in female but not male adolescents born at low birth weight in the offspring of a national cohort Can J Psychiatry 2010; 55(7): 422-30. 